\title{
$\mathrm{Fe}$ 增强 $\mathrm{Ni}_{2}\left(\mathrm{CO}_{3}\right)(\mathrm{OH})_{2}$ 臭氧分解抗湿性与催化性能
}

\author{
黎邦金金 ${ }^{1,2}$, 张 骞 $^{2}$, 肖 杰 $^{2}$, 肖文艳 ${ }^{2}$, 周 莹 ${ }^{1,2}$
}

(西南石油大学 1. 油气藏地质及开发工程国家重点实验室; 2. 材料科学与工程学院 新能源材料及技术研究中心, 成都 610500)

摘 要: 臭氧污染已成为我国继 PM2.5 之后的主要污染物, 传统的臭氧分解催化材料在湿润环境中性能不稳定。本 工作采用水热法制备了一种铁掺杂改性的碱式碳酸镍催化剂( $\mathrm{NiCH}-\mathrm{Fe})$, 该催化剂可在 $60 \%$ 相对湿度下稳定分解 $2.14 \mu \mathrm{g} / \mathrm{L}$ 臭氧 $12 \mathrm{~h}$, 去除率达 $99 \%$ 。水分子吸附质量检测结果表明, $\mathrm{NiCH}-\mathrm{Fe}$ 表面的水分子吸附量比纯 $\mathrm{NiCH}$ 明显 减少, 表面水分子吸脱附可逆; 密度泛函理论结果证明, $\mathrm{Fe}$ 原子是新的代替 $\mathrm{Ni}$ 原子的臭氧吸附位点, 并且 $\mathrm{Fe}$ 原子 对臭氧分子的吸附能力更强。此外, XPS 表明作为活性位点的 Fe 元素在反应前后保持稳定。掺杂 $\mathrm{Fe}$ 后的催化材料 具有优异的抗湿性与长效稳定性。本研究表明通过金属离子掺杂，改变催化材料的表面性质，可获得具有良好抗湿 性能的臭氧催化分解材料，这为进一步开发兼具高抗湿性和高活性的臭氧催化分解材料，提供了有效的技术方法。

关 键 词: 碱式碳酸镍; 掺杂; 臭氧催化分解; 抗湿性

中图分类号: TQ174 文献标志码: A

\section{Iron-doping Enhanced Basic Nickel Carbonate for Moisture Resistance and Catalytic Performance of Ozone Decomposition}

\author{
LI Bangxin ${ }^{1,2}$, ZHANG Qian², XIAO Jie ${ }^{2}$, XIAO Wenyan ${ }^{2}$, ZHOU Ying ${ }^{1,2}$
}

(1. State Key Laboratory of Oil and Gas Reservoir Geology and Exploitation, Southwest Petroleum University, Chengdu 610500, China; 2. The Center of New Energy Materials and Technology, School of Materials Science and Engineering, Southwest Petroleum University, Chengdu 610500, China)

\begin{abstract}
Ozone pollution is taking more dominant position in China than PM2.5, traditional ozonolysis catalytic materials have limited performance in humid conditions. In this study, an iron-doped basic nickel carbonate catalyst (NiCH-Fe) was successfully fabricated via a facile hydrothermal method, which could stably decompose $2.14 \mu \mathrm{g} / \mathrm{L}$ ozone at $60 \%$ relative humidity for $12 \mathrm{~h}$ with nearly $100 \%$ removal ratio. The result of the Quartz Crystal Microbalance test showed that the water molecules adsorbed on the surface of $\mathrm{NiCH}-\mathrm{Fe}$ were significantly reduced as compared with that adsorbed on pure $\mathrm{NiCH}$, which were favorable for the competitive adsorption of ozone. Density functional theory results proved that $\mathrm{Fe}$ atoms were new sites instead of $\mathrm{Ni}$ atoms and had stronger adsorption capacity for ozone molecules. In addition, the XPS results demonstrated that the iron atoms serving as active sites were substantially stable in the reaction. Therefore, material doped with Fe provided excellent moisture resistance and long-term stability. This work provides an effective technical method for the development of
\end{abstract}

收稿日期: 2021-03-04; 收到修改稿日期：2021-05-01; 网络出版日期: 2021-05-25

基金项目：四川省重大科技专项(2020ZDZX0008); 四川省重点研发项目(2021YFSY0046)

Science and Technology Major Projects Sichuan (2020ZDZX0008); Key R\&D Program of Sichuan (2021YFSY0046)

作者简介: 黎邦金金(1995-), 男, 硕士研究生. E-mail: 1449569638@qq.com

LI Bangxin(1995-), male, Master candidate. E-mail: 1449569638@qq.com

通信作者：张 寒，副教授. E-mail: zhangqian@swpu.edu.cn

ZHANG Qian, associate professor. E-mail: zhangqian@swpu.edu.cn 
materials with high moisture resistance ability for efficient ozone catalytic decomposition.

Key words: basic nickel carbonate; doping; ozone decomposition; moisture resistance

近年来, 随着工业发展与汽车保有量的逐步提 高、化石燃料大量燃放以及溶剂的大量使用，挥发 性有机物和氮氧化物的排放不断增加, 臭氧已经成 为我国环境空气污染的首要污染物之一 ${ }^{[1]}$ 。与室外 环境相比, 室内环境中的臭氧污染更为严重: 家用 电器、办公电器、空气净化器等在使用过程中均会 产生臭氧。臭氧不但会造成呼吸系统、视觉系统的 永久损伤, 而且会与室内环境中的其他气体发生反 应, 加重室内污染 ${ }^{[2-3]}$ 。虽然, 近年来空气中挥发性 有机物和氮氧化物均得到了有效的控制, 对应的催 化材料研究也逐步完善 ${ }^{[4-6]}$, 但环境中的臭氧浓度 却呈现逐年增长的趋势 ${ }^{[7]}$ 。臭氧作为一种强氧化剂, 可以长期留存在近地面与室内环境中, 时刻威胁 着人体的健康, 因此净化环境中的臭氧污染已刻 不容缓。

常温催化分解法是臭氧污染治理方法中条件温 和且节能环保的处理手段之一, 常用的催化材料有 贵金属 ${ }^{[8]}$ 、过渡金属氧化物 ${ }^{[9]}$ 、固体金属盐 ${ }^{[10]}$ 等。 但是臭氧污染气体中存在远高于臭氧浓度的水分子, 由于它与臭氧分子竞争活性位, 使臭氧在材料表面 的吸附和反应位点的减少, 导致催化材料臭氧分解 性能快速降低, 存在严重的抗湿性问题 ${ }^{[11]}$ 。针对上 述问题, 已有研究表明, 对传统的锰基催化材料进 行掺杂 ${ }^{[12]}$ 、复合 ${ }^{[13]}$ 和晶格调控 ${ }^{[14]}$ 能够通过提升催化 材料位点的臭氧吸附性能或是减弱水分子的吸附, 有 效地提升催化材料抗湿性能。镍基材料具有高效催 化臭氧分解的性能 ${ }^{[15]}$, 也同样存在抗湿性问题, 但少 有学者针对此类材料的抗湿性能的提升开展研究。

鉴于此, 本工作选用镍基催化材料碱式碳酸镍 $\mathrm{Ni}_{2}\left(\mathrm{CO}_{3}\right)(\mathrm{OH})_{2}$ 进行实验, 通过对这种具有抗湿问题 的材料进行金属离子掺杂, 改变材料的表面组成, 使材料在湿润环境中获得稳定的臭氧催化分解能力。

\section{1 实验方法}

\section{1 催化材料的制备}

采用水热法制备碱式碳酸镍。首先, 配置 $\mathrm{NiCl}_{2} \cdot 6 \mathrm{H}_{2} \mathrm{O}(0.006 \mathrm{~mol}) 、 \mathrm{FeCl}_{3}(\mathrm{Ni}: \mathrm{Fe}=100: x$, 摩尔 比)、尿素 $(0.024 \mathrm{~mol}) 、 480 \mathrm{~mL}$ 的去离子水溶液; 然 后，在聚四氟乙烯高温反应釜中 $120{ }^{\circ} \mathrm{C}$ 下水热反应 $16 \mathrm{~h}$; 抽滤洗涤(去离子水、乙醇)后, 在 $120{ }^{\circ} \mathrm{C}$ 下干 燥 $12 \mathrm{~h}$, 得到相应的 $x \% \mathrm{Fe}$ 掺杂量的碱式碳酸镍
(NiCH-Fe $x \%$ )催化材料。水热溶液中未加入 $\mathrm{FeCl}_{3}$ 或 $\mathrm{NiCl}_{2} \cdot 6 \mathrm{H}_{2} \mathrm{O}$, 以制备得到碱式碳酸铁 $(\mathrm{FeCH})$ 、碱 式碳酸镍 $(\mathrm{NiCH})$ 样品。所有实验试剂均为分析纯, 采购自阿拉丁。

\section{2 材料表征}

采用 $\chi$ 'Pert PRO MPD 型 X 射线衍射仪, 测试电 压 $40 \mathrm{kV}$, 射线源为 $\mathrm{Cu} \mathrm{k} \alpha$; ZEISS EV0 MA15 扫描电 子显微镜, 电压为 $20 \mathrm{kV}$; FEI Tecnai G2 F20 透射电子 显微镜; ASAP 2460 比表面与孔隙度分析仪, $77.3 \mathrm{~K}$; ESCALAB 250Xi 光电子能谱仪分别对催化材料的结 构、形貌、比表面积以及反应前后的结合能进行测试。

利用石英晶体微天平 $(\mathrm{QCM})$ 测量材料表面吸附 水分子的质量 ${ }^{[16]}$ 。晶片基频为 $500 \mathrm{kHz}$, 采用乙醇配 置 $0.01 \mathrm{~g} / \mathrm{mL}$ 的材料悬浊液, 取 $20 \mu \mathrm{L}$ 分散液, 在 $60{ }^{\circ} \mathrm{C}$ 环境下干燥 $24 \mathrm{~h}$ 。为排除氧气的影响, 实验气体选 用氮气, 配置干燥和相对湿度 $60 \%$ 的两种气体用于 测试, 经晶片表面的气流流速固定为 $5 \mathrm{~mL} / \mathrm{min}$ 。

\section{3 性能测试}

使用固定床反应器测试材料的臭氧分解性能。臭 氧由真空紫外 $(185 \mathrm{~nm})$ 光源产生, 浓度为 $(40 \pm 2) \mathrm{ppm}$ $((2.14 \pm 0.1) \mu \mathrm{g} / \mathrm{L})$, 固定床管径 $\phi 8 \mathrm{~mm}$, 催化材料篮 选 80 目 $(187.5 \mu \mathrm{m})$, 用量 $100 \mathrm{mg}$, 混合 $300 \mathrm{mg}$ 石英 砂; 反应温度控制在 $25^{\circ} \mathrm{C}$, 经过装置的气流总流量 为 $1.5 \mathrm{~L} / \mathrm{min}$, 湿度通过鼓泡装置控制。利用 Model 106L(2B Technologies)检测臭氧浓度, 利用 GSP-6 (精创)检测温湿度。

\section{4 理论计算}

利用 Cambridge Sequential Total Energy Package(CASTEP) 程序进行密度泛函理论(DFT) 计 算 ${ }^{[17]}$, 采用广义梯度近似(GGA/PBE)泛函说明交换 关联能, 截断能设置为 $380 \mathrm{eV}$ 。在所有的计算中布 里渊区采用的是 $2 \times 2 \times 1$ 的 $\mathrm{k}$ 点。几何结构优化均在 所有原子完全松弛下进行, 通过构建 $1.5 \mathrm{~nm}$ 的真空层 以避免周期性结构之间的相互作用，以 $0.005 \mathrm{eV} / \mathrm{nm}$ 和 $1 \times 10^{-5} \mathrm{eV}$ 阈值进行了优化。材料结构的吸附能 $\left(E_{\mathrm{ad}}\right)$ 通过以下公式计算:

$$
E_{\text {ad }}=E_{\text {(composite }+ \text { adsorbate) }}-E_{\text {composite }}-E_{\text {adsorbate }}
$$

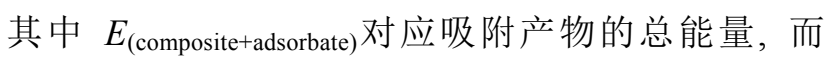
$E_{\text {composite }}$ 与 $E_{\text {adsorbate }}$ 分别对应其中的材料与相应的吸 附物 $\mathrm{O}_{3}$ 的能量。 


\section{2 结果与讨论}

\section{1 结构分析与表征}

图 1 为 $\mathrm{NiCH}$ 与 $\mathrm{NiCH}-\mathrm{Fe}$ 的 XRD 图谱, 由图可 见, $\mathrm{Fe}$ 掺杂催化材料均存在与 PDF\#40-0215 标准卡 片相对应的特征峰, 表明掺杂的催化材料中形成了 碱式碳酸铁镍 $\left(\mathrm{Ni}_{0.75} \mathrm{Fe}_{0.25}\left(\mathrm{CO}_{3}\right)_{0.125}(\mathrm{OH})_{2} \cdot 0.38 \mathrm{H}_{2} \mathrm{O}\right)$, 随着 $\mathrm{Fe}$ 掺杂量的增加, 对应的特征峰强度明显增强, 特别是在掺杂 $20 \% \mathrm{Fe}$ 的样品中观察到了属于 $\mathrm{Ni}\left(\mathrm{HCO}_{3}\right)_{2}$ 的特征峰(绿色三角标记), 而未掺杂 $\mathrm{Fe}$ 离子的催化材料无明显的特征峰, 这可能是由于 $\mathrm{Fe}$ 离子改变了水热前驱溶液的 $\mathrm{pH}$ 造成的 ${ }^{[18]}$ 。根据相关 文献报道 ${ }^{[19]}$, 合成得到的碱式碳酸镍 $\mathrm{Ni}_{2}\left(\mathrm{CO}_{3}\right)(\mathrm{OH})_{2}$ 为无定型材料。上述 XRD 数据表明 $\mathrm{Fe}$ 的掺杂能改 变碱式碳酸镍的晶型, 得到掺杂有 $\mathrm{Fe}$ 元素的碱式碳 酸铁镍, 并且随着铁离子掺入量的增加, 低结晶度 的碱式碳酸镍向高结晶度的碱式碳酸铁镍与碳酸氢 镍转化。

通过扫描电子显微镜观察 $\mathrm{NiCH}-\mathrm{Fe} 5 \%$ 样品的 表面形貌, 如图 2(a)所示, NiCH-Fe5\%样品表面覆盖 着一层线毛状物质, 这与碱式碳酸镍形貌一致 ${ }^{[20]}$ 。

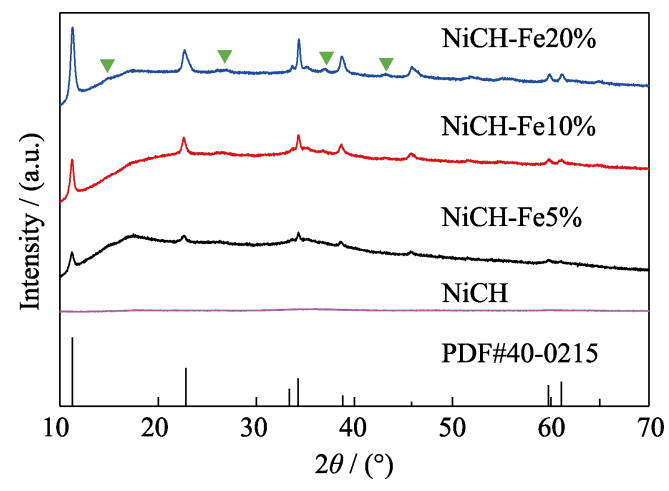

图 $1 \mathrm{NiCH}$ 与 $\mathrm{NiCH}-\mathrm{Fe}$ 的 XRD 图谱

Fig. 1 XRD patterns of $\mathrm{NiCH}$ and $\mathrm{NiCH}-\mathrm{Fe}$

EDS 元素分析结果显示, 样品表面均匀分布着 $\mathrm{Ni}$ (图 2(b)) 与 $\mathrm{Fe}$ (图 2(c)) 元素。对比 $\mathrm{NiCH}$ (图 2(d)) 与 NiCH- Fe 5\%(图 2(e))的 TEM 照片可发现，掺杂前后 的材料均由片层结构堆叠而成, 但未掺杂的 $\mathrm{NiCH}$ 结构致密, 仅边缘能够观察到片层状结构。 $\mathrm{NiCH}-\mathrm{Fe} 5 \%$ 微观片层结构清晰, 颗粒尺寸小于未掺 杂 $\mathrm{Fe}$ 的样品, NiCH-Fe5\%的高分辨 TEM 照片(图 2(f)) 显示出对应碱式碳酸镍与碱式碳酸铁镍的两种晶格 条纹, 这与 XRD 分析结果一致。对比 SEM 与 TEM
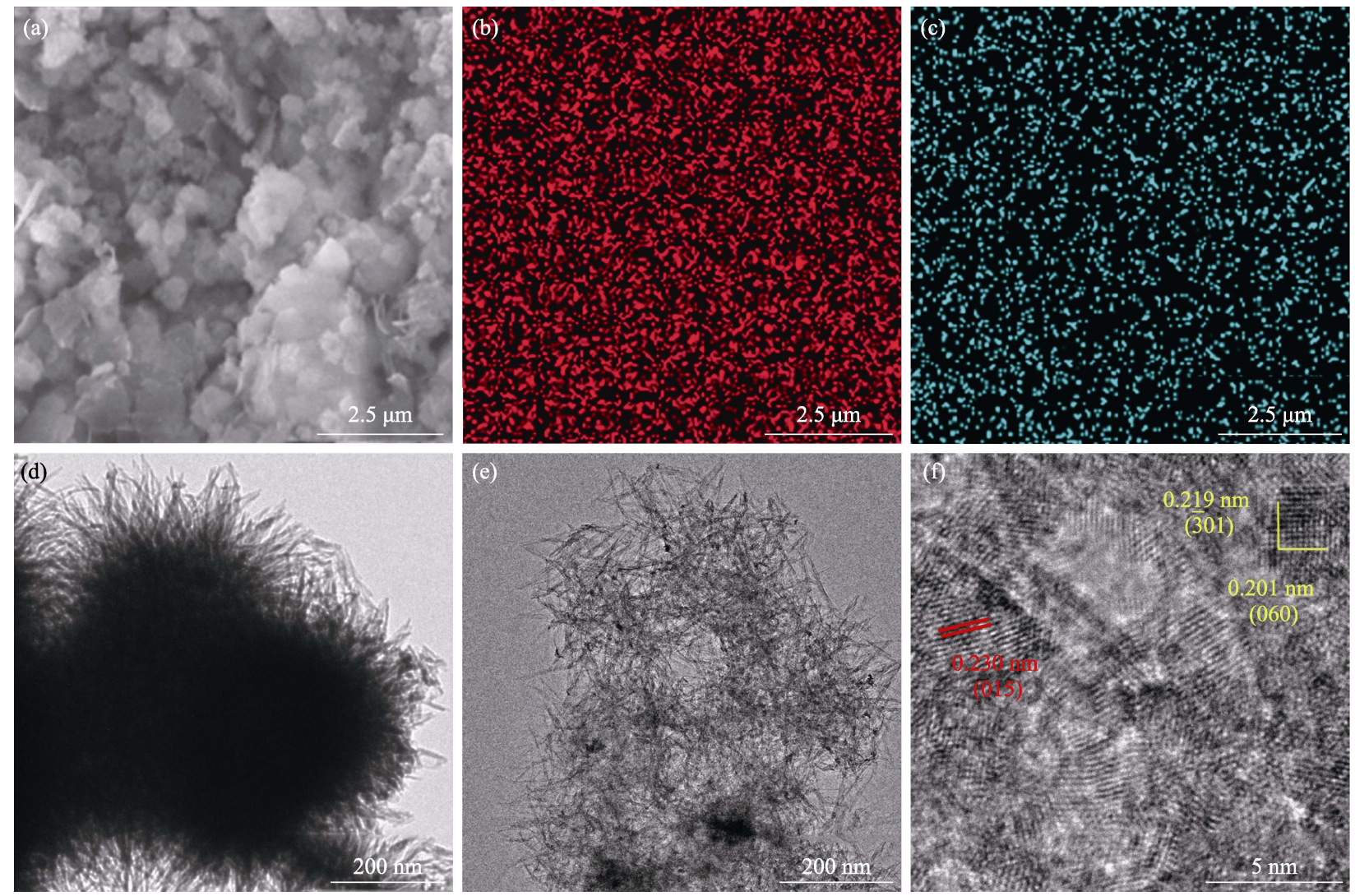

图 $2 \mathrm{NiCH}-\mathrm{Fe} 5 \%$ 样品的(a)SEM 照片及其上 $\mathrm{Ni}(\mathrm{b}) 、 \mathrm{Fe}$ (c)元素的 EDS 分布; (d) $\mathrm{NiCH}$ 和 (e) NiCH-Fe5\%的 TEM 照片; (f) NiCH-Fe5\%的 HRTEM 照片

Fig. 2 (a) SEM image of NiCH-Fe $5 \%$ and corresponding EDS mappings of (b) Ni and (c) Fe elements, TEM images of (d) $\mathrm{NiCH}$ and (e) $\mathrm{NiCH}-\mathrm{Fe} 5 \%$, and (f) HRTEM image of $\mathrm{NiCH}-\mathrm{Fe} 5 \%$ 
照片, $\mathrm{Fe}$ 掺杂前后的 $\mathrm{NiCH}$ 样品表面微观形貌相近, 但掺杂后样品的颗粒尺寸明显减小, 这可能是材料 比表面积增加的原因。 $\mathrm{Fe}$ 与 $\mathrm{Ni}$ 在材料表面的分布 均匀, 说明 $\mathrm{Fe}$ 元素成功进入了材料的晶格, 使材料掺 杂后拥有不同的反应活性位点。如图 3(a)所示, 材 料 $\mathrm{N}_{2}$ 吸脱附数据表明掺杂 $\mathrm{Fe}$ 对催化材料的比表面 积影响明显。掺杂少量 $\mathrm{Fe}$ 可以显著提升材料的表面 积, 而过量掺杂 $\mathrm{Fe}$ 则会使催化材料的比表面积急剧 降低, 这可能是 $\mathrm{Fe}$ 过量掺杂引起材料结晶度升高以 及晶型转化造成的。同时, 图 3(b)材料的孔径分布 趋势除 $\mathrm{FeCH}$ 外无明显变化。因此, 掺杂适量 $\mathrm{Fe}$ 能 够明显提升催化材料的比表面积, 并为臭氧催化分 解提供更多的反应位点。

\section{2 臭氧分解性能测试}

制备材料的臭氧分解性能测试结果如图 4(a)所 示。不同 $\mathrm{Fe}$ 掺杂量的催化材料在 $60 \%$ 的相对湿度 下表现出不同的臭氧分解性能。未掺杂 $\mathrm{Fe}$ 的 $\mathrm{NiCH}$ 材料反应 $30 \mathrm{~min}$ 后臭氧分解性能下降至 $90 \%$, 而掺 杂量为摩尔分数 $1 \% 、 10 \%$ 的 $\mathrm{NiCH}$ 材料臭氧分解性 能稳定在 $96 \%$ 左右。 $\mathrm{NiCH}-\mathrm{Fe} 20 \%$ 在反应开始 $3 \mathrm{~min}$ 内性能下降明显，之后在测试时间内恢复到 $95 \%$ 。 5 个样品中仅 $\mathrm{NiCH}-\mathrm{Fe} 5 \%$ 材料在测试时间内维持了 $100 \%$ 的臭氧分解效率。
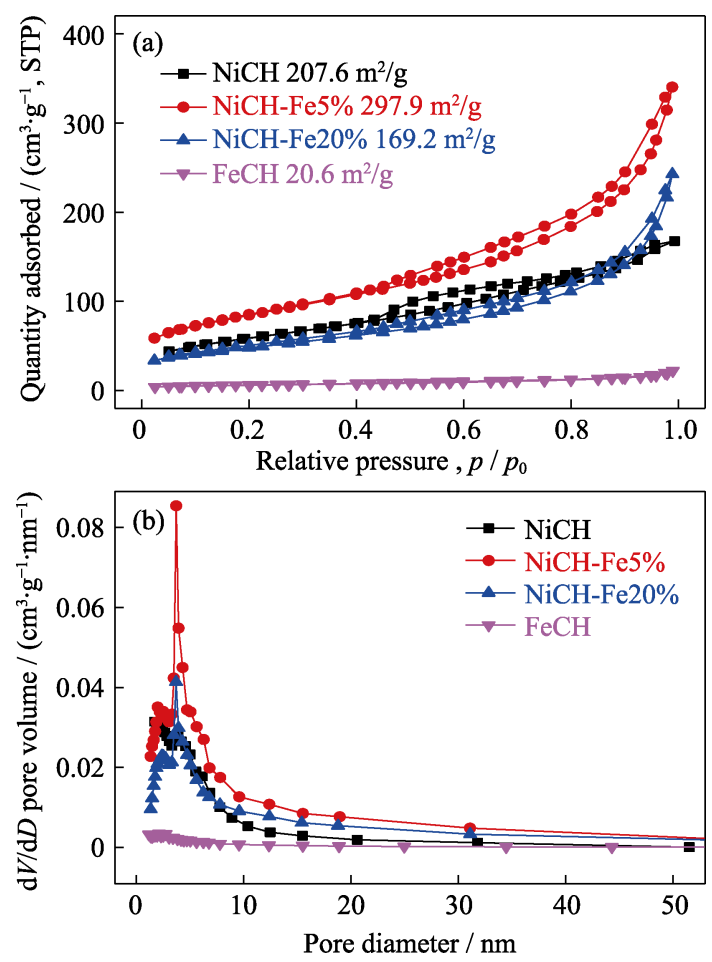

图 $3 \mathrm{NiCH} 、 \mathrm{NiCH}-\mathrm{Fe} 、 \mathrm{FeCH}$ 的(a)氮气吸脱附等温曲线与 (b)孔径分布图

Fig. 3 (a) Nitrogen adsorption-desorption isotherms and (b) pore size distributions of $\mathrm{NiCH}, \mathrm{NiCH}-\mathrm{Fe}$ and $\mathrm{FeCH}$
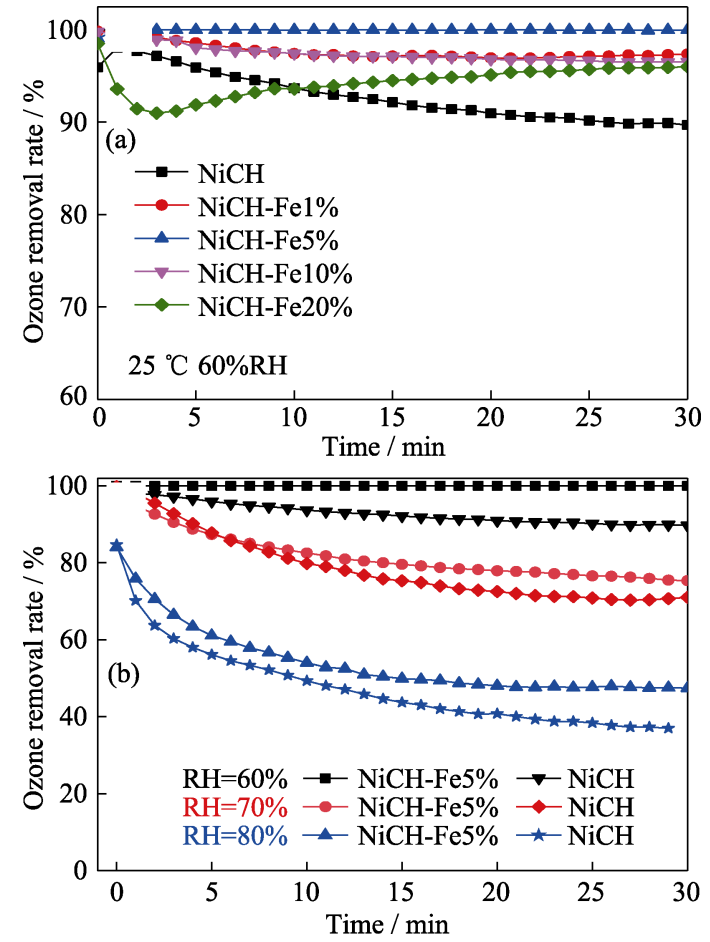

图 4 (a)不同铁掺杂量的 $\mathrm{NiCH}-\mathrm{Fe}$ 在 $60 \%$ 相对湿度下与 (b) NiCH-Fe5\%材料在 $60 \% 、 70 \% 、 80 \%$ 相对湿度下的臭氧去 除率

Fig. 4 Ozone removal rates of (a) NiCH-Fe with different $\mathrm{Fe}$ dopants at $60 \% \mathrm{RH}$ and (b) NiCH-Fe $5 \%$ at $60 \%, 70 \%, 80 \% \mathrm{RH}$

基于以上测试结果，进一步探究 $\mathrm{NiCH}-\mathrm{Fe} 5 \%$ 的 抗湿性能, 测试结果如图 4(b)表示。 NiCH-Fe5\%催 化材料在相对湿度高于 $60 \%$ 的条件下，催化性能受 湿度影响明显, 随着湿度增加, $\mathrm{NiCH}-\mathrm{Fe} 5 \%$ 臭氧分 解性能明显下降, 在 $80 \%$ 的湿度下臭氧分解性能仅 能稳定在 $50 \%$, 但较未掺杂材料均有提升。以上结 果表明, 相较于未掺杂的碱式碳酸镍, 掺杂 $\mathrm{Fe}$ 可以 较好地改善催化材料的抗湿性能。长时间臭氧分解 性能测试结果表明，掺杂 $\mathrm{Fe}$ 的催化材料不仅具有较 好的抗湿性能, 而且还表现出较好的长效稳定性, 可在较长的时间内保持 $99 \%$ 的臭氧分解性能，远高 于商业 $\mathrm{MnO}_{2}$ (图 5)。

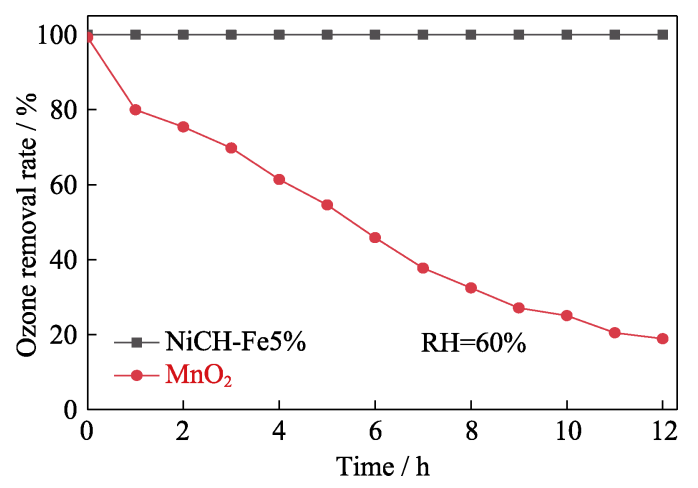

图 $5 \mathrm{NiCH}-\mathrm{Fe} 5 \%$ 与 $\mathrm{MnO}_{2}$ 的长时间臭氧分解性能测试

Fig. 5 Long time ozone removal tests of $\mathrm{NiCH}-\mathrm{Fe} 5 \%$ and $\mathrm{MnO}_{2}$ 


\section{3 催化材料表面性质}

通过 XPS 检测催化材料反应前后元素价态的变 化。NiCH-Fe $5 \%$ 中 $\mathrm{Ni}$ 元素的 $2 \mathrm{p}_{3 / 2}$ 结合能为 $857.0 \mathrm{eV}$, 以 $\mathrm{Ni}^{2+}$ 的形式存在 ${ }^{[21]} ; \mathrm{Fe}$ 元素 $2 \mathrm{p}_{3 / 2}$ 的结合能为 $713.4 \mathrm{eV}$, 对应 $\mathrm{Fe}(\mathrm{III})^{[22]}$ 。对比反应前后 $\mathrm{NiCH}-\mathrm{Fe} 5 \%$ 中 $\mathrm{Fe} 、 \mathrm{Ni}$ 的结合能发现, $\mathrm{NiCH}-\mathrm{Fe} 5 \%$ 与臭氧反应使 其中 $\mathrm{Ni}$ 的结合能提高了 $0.68 \mathrm{eV}$ (图 6(a)), 但 $\mathrm{Fe}$ 元 素的结合能无明显变化(图 6(b))。这表明材料中的 $\mathrm{Ni}$ 原子被臭氧氧化 ${ }^{[23]}$, 反应前后可逆性差, 而掺杂 的 $\mathrm{Fe}$ 价态稳定。 $\mathrm{Ni}$ 材料元素价态变化往往会引起 材料晶体结构变化, 从而导致材料催化活性改变 ${ }^{[24]}$ 。 掺杂 $\mathrm{Fe}$ 元素提高了镍基臭氧催化分解材料的结构稳 定性，使 $\mathrm{NiCH}-\mathrm{Fe} 5 \%$ 性能达到长效稳定。

材料的抗湿性与材料表面对水分子的吸附性密 切相关，通过 QCM 检测了掺杂前后材料表面的水 分子吸附情况 ${ }^{[16]}$, 如图 7 所示。 $\mathrm{NiCH}-\mathrm{Fe} 5 \%$ 在 $60 \%$ 相对湿度下水分子的吸附量较未掺杂材料明显减少, 仅为 $0.03 \mathrm{~g} / \mathrm{g}$ 的吸附量。材料表面水分子均可在干 燥气流中脱除, 说明水分子在材料表面的吸附是可 逆的。以上实验结果表明, 掺杂 $\mathrm{Fe}$ 可以明显降低催 化材料表面水分子的吸附量, 因此在湿润环境中 $\mathrm{NiCH}-\mathrm{Fe} 5 \%$ 表现出更好的抗湿性与催化稳定性。

\section{4 理论计算}

依据材料的晶体结构参数, 建立了 $\mathrm{NiCH}-\mathrm{Fe}$ 理
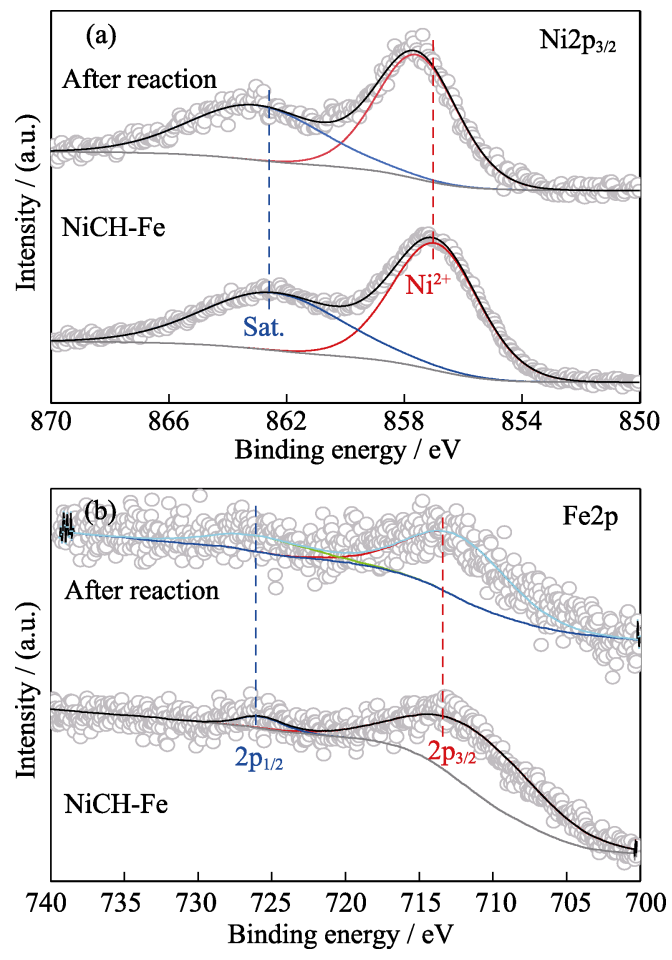

图 $6 \mathrm{NiCH}-\mathrm{Fe} 5 \%$ 反应前后的(a)Ni2 $\mathrm{p}_{3 / 2}$ 和(b)Fe $2 \mathrm{p}$ 的 XPS 图谱

Fig. 6 XPS spectra of (a) Ni2 $\mathrm{p}_{3 / 2}$ and (b) $\mathrm{Fe} 2 \mathrm{p}$ of $\mathrm{NiCH}-\mathrm{Fe} 5 \%$ before and after reaction

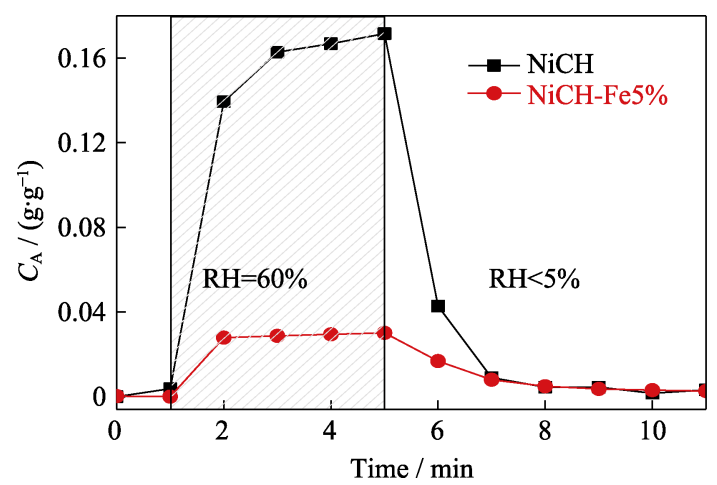

图 $7 \mathrm{NiCH}$ 与 $\mathrm{NiCH}-\mathrm{Fe} 5 \%$ 材料表面水分子吸附曲线

Fig. 7 Surface water adsorption curves of $\mathrm{NiCH}$ and $\mathrm{NiCH}-$ $\mathrm{Fe} 5 \%$

论计算模型，用于计算表面臭氧吸附性质。由 XRD 数据可以获取如图 8 所示模型, 臭氧的可能吸附位 点标记为 $\mathrm{Fe} 、 \mathrm{O} 1 、 \mathrm{O} 2 、 \mathrm{Ni}$ ，未掺杂的样品中以 $\mathrm{Ni}$ 替换 $\mathrm{Fe}$ 位点。

计算得到的臭氧与水分子的吸附能结果如表 1 所示。对比 $\mathrm{NiCH}$ 与 $\mathrm{NiCH}-\mathrm{Fe}$ 上不同位点臭氧的吸 附能, 可以发现臭氧在未掺杂材料中活性位点 $\mathrm{Ni}$ 原 子上的吸附能较负, 表明未掺杂催化材料中 $\mathrm{Ni}$ 为催 化臭氧分解反应中的吸附位点 ${ }^{[25]}$; 而掺杂 $\mathrm{Fe}$ 后, $\mathrm{NiCH}-\mathrm{Fe}$ 表面 $\mathrm{Fe}$ 位点的吸附能较 $\mathrm{Ni}$ 更负, 为 $-1.93 \mathrm{eV}$, 表明臭氧更容易吸附在掺杂后 $\mathrm{NiCH}-\mathrm{Fe}$ 材料的 $\mathrm{Fe}$

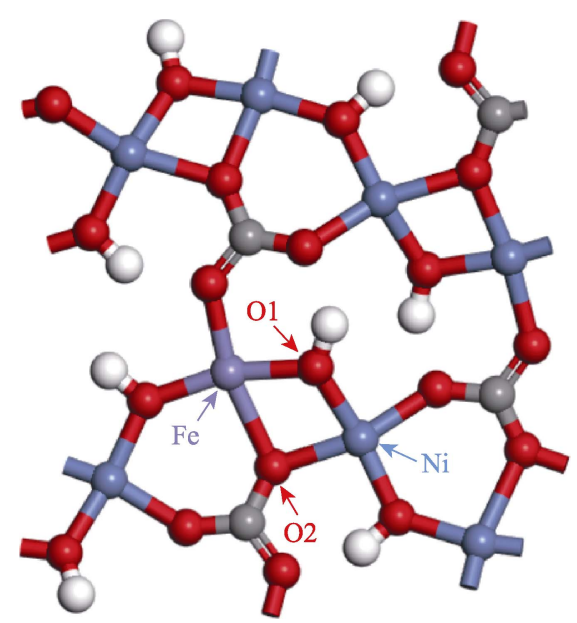

图 8 单层的 $\mathrm{NiCH}-\mathrm{Fe}$ 的模型

Fig. 8 Single-layer structure of $\mathrm{NiCH}-\mathrm{Fe}$

表 $1 \mathrm{NiCH}$ 与 NiCH-Fe 上不同位点臭氧与水分子的吸附能 Table 1 Adsorption energy of ozone and $\mathrm{H}_{2} \mathrm{O}$ on different sites of $\mathrm{NiCH}$ and NiCH-Fe5\%

\begin{tabular}{ccll}
\hline Molecule & Site & \multicolumn{1}{c}{$\mathrm{NiCH}$} & \multicolumn{1}{l}{ NiCH-Fe } \\
\hline & $\mathrm{Ni}$ & $-0.36 \mathrm{eV}$ & $-0.57 \mathrm{eV}$ \\
$\mathrm{O}_{3}$ & $\mathrm{Fe}(\mathrm{Ni})$ & $-0.02 \mathrm{eV}(\mathrm{Ni})$ & $-1.93 \mathrm{eV}(\mathrm{Fe})$ \\
& $\mathrm{O} 1$ & $-0.28 \mathrm{eV}$ & $-0.61 \mathrm{eV}$ \\
& $\mathrm{O} 2$ & $-0.69 \mathrm{eV}$ & $-0.46 \mathrm{eV}$ \\
\hline \multirow{2}{*}{$\mathrm{H}_{2} \mathrm{O}$} & $\mathrm{Ni}$ & $-0.15 \mathrm{eV}$ & $-0.41 \mathrm{eV}$ \\
& $\mathrm{Fe}(\mathrm{Ni})$ & $-0.24 \mathrm{eV}(\mathrm{Ni})$ & $-0.55 \mathrm{eV}(\mathrm{Fe})$ \\
\hline
\end{tabular}


原子上。对比位点上水分子吸附能的改变, 掺杂 $\mathrm{Fe}$ 后材料对臭氧吸附能力的提升远大于水分子, 竞争 吸附中臭氧依然占据主要地位。因此, $\mathrm{Fe}$ 元素的掺 杂不但丰富了材料的臭氧吸附活性位点，而且增强 了材料对臭氧的吸附捕获能力, 有利于进一步提升 催化材料催化分解臭氧的能力。

\section{3 结论}

掺杂 $\mathrm{Fe}$ 元素可以有效提升碱式碳酸镍对臭氧的 分解能力, 提高了在臭氧分解反应过程中反应位点 的价态稳定性，并通过增强材料表面吸附臭氧的能 力，使其反应中水分子吸附量减少，最终获得能够 在 $60 \%$ 相对湿度的环境中稳定去除臭氧的催化材料 $\mathrm{NiCH}-\mathrm{Fe} 5 \%$ 。本实验分析了掺杂元素如何改变催化 材料的性质, 从吸附原理方面探究了催化材料抗湿 性能提升的原因, 促进了对现有催化材料抗湿性能 稳步提升问题的研究, 同时也为新型抗湿性臭氧催 化材料的研发提供了方案。

\section{参考文献:}

[1] MULLiNS J T. Ambient air pollution and human performance: contemporaneous and acclimatization effects of ozone exposure on athletic performance. Health Economics, 2018, 27(8): 1189-1200.

[2] FU P F, CHEN S J. Indoor air pollution caused by ozone reactions and problems in disinfection and purification by using ozone air cleaners. Journal of Beijing Union University (Natural Sciences), 2006, 20(3): 73-75.

[3] SHAO G M. The hazards of ozone pollution and protective measure in copy room. Contamination Control Air conditioning Technology, 2017, 02: 80-83.

[4] WU F, ZHAO Z Y, LI B X et al. Interfacial oxygen vacancy of $\mathrm{Bi}_{2} \mathrm{O}_{2} \mathrm{CO}_{3} / \mathrm{PPy}$ and its visible-light photocatalytic NO oxidation mechanism. Journal of Inorganic Materials, 2020, 35(5): 541-548.

[5] ZHANG R Y, LI C J, ZHANG A L, et al. Research progress on the preparation and application of monolithic photocatalysts. Materials Reports, 2020, 34(3): 3001-3016.

[6] CAO Y H, ZHENG Q, RAO Z Q, et al. InP quantum dots on g- $\mathrm{C}_{3} \mathrm{~N}_{4}$ nanosheets to promote molecular oxygen activation under visible light. Chinese Chemical Letters, 2020, 32(10): 2689-2692.

[7] WANG T, XUE L K, BRIMBLECOMBE P, et al. Ozone pollution in China: a review of concentrations, meteorological influences, chemical precursors, and effects. Science of The Total Environment, 2017, 575: 1582-1596.

[8] LI X T, MA J Z, HE H. Tuning the chemical state of silver on Ag-Mn catalysts to enhance the ozone decomposition performance. Environmental Science \& Technology, 2020, 54(18): 11566-11575.

[9] DHANDAPANI B, OYAMA S T. Gas phase ozone decomposition catalysts. Applied Catalysis B: Environmental, 1997, 11(2): 129-166.
[10] JIA J B, ZHANG P Y. Catalytic decomposition of airborne ozone by $\mathrm{MnCO}_{3}$ and its mechanism. Ozone: Science \& Engineering, 2018, 40(1): 21-28.

[11] LIAN Z H, MA J Z, HE H. Decomposition of high-level ozone under high humidity over Mn-Fe catalyst: The influence of iron precursors. Catalysis Communications, 2015, 59: 156-160.

[12] YANG Y J, ZHANG P Y, JIA J B. Vanadium-doped $\mathrm{MnO}_{2}$ for efficient room-temperature catalytic decomposition of ozone in air. Applied Surface Science, 2019, 484: 45-53.

[13] GONG S Y, CHEN J Y, WU X F, et al. In-situ synthesis of $\mathrm{Cu}_{2} \mathrm{O}$ /reduced graphene oxide composite as effective catalyst for ozone decomposition. Catalysis Communications, 2018, 106: 25-29.

[14] JIA J B, ZHANG P Y, CHEN L. Catalytic decomposition of gaseous ozone over manganese dioxides with different crystal structures. Applied Catalysis B: Environmental, 2016, 189: 210-218.

[15] YU H F. Catalytic decompostion of ozone over monolithic catalysts with deferect ninch salt precursors. Journal of Qingdao University of Science and Technology (Natural Science Edition), 2019, 40(6): 27-30.

[16] SPINELLA K, MOSIELLO L, PALLESCHI G, et al. Development of a QCM (Quartz Crystal Microbalance) biosensor to the detection of aflatoxin B1. Open Journal of Applied Biosensor, 2013, 2(4): 112-119.

[17] CLARK S J, SEGALL M D, PICKARD C J, et al. First principles methods using CASTEP. Zeitschrift für Kristallographie-Crystalline Materials, 2005, 220(5/6): 567-570.

[18] DAI S G, ZHANG Z F, XU J M, et al. In situ Raman study of nickel bicarbonate for high-performance energy storage device. Nano Energy, 2019, 64: 103919-1-9.

[19] YU Z M, SU X L, WEI D H, et al. Tiny basic nickel carbonate arrays/reduced graphene oxide composite for high-efficiency supercapacitor application. Nano, 2019, 14(4): 96-103.

[20] XI C Y, ZHU G X, LIU Y J, et al. Belt-like nickel hydroxide carbonate/ reduced graphene oxide hybrids: synthesis and performance as supercapacitor electrodes. Colloids and Surfaces A: Physicochemical and Engineering Aspects, 2018, 538: 748-756.

[21] BIESINGER M C, PAYNE B P, LAU L W M, et al. X-ray photoelectron spectroscopic chemical state quantification of mixed nickel metal, oxide and hydroxide systems. Surface and Interface Analysis, 2009, 41(4): 324-332.

[22] YAMASHITA T, HAYES P. Analysis of XPS spectra of $\mathrm{Fe}^{2+}$ and $\mathrm{Fe}^{3+}$ ions in oxide materials. Applied Surface Science, 2008, 254(8): 2441-2449.

[23] STOYANOVA M, KONOVA P, NIKOLOV P, et al. Aluminasupported nickel oxide for ozone decomposition and catalytic ozonation of CO and VOCs. Chemical Engineering Journal, 2006, 122(1/2): 41-46.

[24] HU C W, YAMADA Y, YOSHIMURA K. Fabrication of nickel oxyhydroxide/palladium (NiOOH/Pd) nanocomposite for gasochromic application. Solar Energy Materials and Solar Cells, 2018, 177: 120-127.

[25] ABBASI A, SARDROODI J J. Application of $\mathrm{TiO}_{2}$-supported $\mathrm{Au}$ for ozone molecule removal from environment: a van der Waalscorrected DFT study. International Journal of Environmental Science and Technology, 2019, 16(7): 3483-3496. 